\title{
Mode of Delivery for a Term Patient with Spontaneous Coronary Artery Dissection
}

\author{
Cheryl Yondorf*, Arnold Cohen \\ Einstein Medical Center Philadelphia, 5501 Old York Road, Philadelphia PA 1914, USA
}

*Corresponding Author: Cheryl Yondorf, Einstein Medical Center Philadelphia, 5501 Old York Road, Philadelphia, Pennsylvania,USA, Email:yondorfc@einstein.edu

\begin{abstract}
Background: Management of spontaneous coronary artery dissection (SCAD) depends on maternal stability and fetal status. When SCAD is diagnosed in the antepartum period, consideration must be given to mode of delivery of the fetus.

Case presentation: 32 -year-old G9P4134 presented at 38.3 weeks gestation with SCAD. She was managed medically and remained stable. With limited data to guide us, the decision for cesarean section was made based on maximizing the resources available to care for a sudden cardiac event during labor or delivery.

Conclusion: We believe from this case that the standard approach to labor and delivery for patients with cardiac disease (vaginal delivery with expedited second stage of labor) may not apply to patients with SCAD.

Keywords:Myocardial infarction, Pregnancy, Delivery, Spontaneous coronary artery dissection

Abbreviations:Spontaneous coronary artery dissection (SCAD), ST elevation myocardial infarction (STEMI), Non-ST elevation myocardial infarction (NSTEMI), coronary artery bypass graft (CABG), percutaneous coronary intervention (PCI)
\end{abstract}

\section{INTRODUCTION}

Among the general population, spontaneous coronary artery dissection (SCAD) is a very uncommon cause of myocardial infarction. However, among the pregnant population, SCAD is the cause of greater than $40 \%$ of myocardial infarctions ${ }^{1}$. It typically affects young women who do not have any of the common risk factors for atherosclerosis ${ }^{2}$. The most common presenting symptom is chest pain, often associated with diaphoresis, nausea and dyspnea. In the Mayo clinic SCAD registry, $49 \%$ of patients presented with ST elevation myocardial infarction (STEMI), 44\% presented with non-ST elevation myocardial infarction (NSTEMI) and $7 \%$ presented with unstable angina $^{3}$. Among pregnant women, the most common time for presentation was in the late third trimester, intrapartum or postpartum ${ }^{4}$.

Management of this condition depends on maternal stability as well as fetal status. If the patient is unstable, an emergent coronary artery bypass graft (CABG) surgery is necessary. However, there is a high rate of fetal morbidity and mortality associated with cardiac bypass ${ }^{5-10}$. When the patient is stable, management options include revascularization via percutaneous coronary intervention (PCI) versus conservative medical management with close monitoring. PCI in this group of patients has been associated with a low success rate and a high incidence of complications, most notably propagation of the existing dissection. Therefore, conservative medical management is preferred ${ }^{4}, 11$ with continued inpatient observation for minimum of five days to monitor for progression of the $\mathrm{SCAD}$ that may require emergent $\mathrm{CABG}{ }^{12}$.

Most of these cases occur in the postpartum period, however when SCAD is diagnosed in the antepartum period, consideration must be given to mode of delivery of the fetus. In this paper, we present a case of SCAD that occurred in the late third trimester where a significant area of discussion in the management focused on the approach for delivery of this patient.

\section{CASE Presentation}

A 32-year-old G9P4134 at 38.3 weeks gestation by last menstrual period consistent with 12.1 week ultrasound presented to the emergency department complaining of acute onset, crushing, substernal chest pain. The pain began that morning and was associated with left arm pain and numbness, diaphoresis, dyspnea, and 
inability to function. The patient denied any alleviating or exacerbating factors. Five days prior to this episode the patient was diagnosed with influenza and was started on Tamiflu. The patient reported improvement in her "flu" symptoms until the morning of presentation.

Her obstetric history was significant for two first-trimester elective terminations, one firsttrimester spontaneous abortion, four full term uncomplicated vaginal deliveries and one preterm delivery at 35 weeks. That infant was found to have Apert syndrome, multiple congenital anomalies, and died shortly after delivery. The patient reported that her last two deliveries occurred precipitously, with barely enough time to get to the hospital.

The patient's medical history was significant for mild intermittent asthma. The patient's surgical history was significant for an umbilical hernia repair. The patient denied any family history of cardiac disease. Her medications included albuterol as needed. She had a history of tobacco and marijuana use but denied any recent use.

On exam, her vital signs were stable with a heart rate of 88 beats per minute, blood pressure of $126 / 59 \mathrm{~mm} \mathrm{Hg}$, and oxygen saturation of $100 \%$ on room air. She was in significant distress, diaphoretic and clutching her chest. Her heart and lung exams were within normal limits. Her abdomen was gravid, soft, non-tender and no contractions were palpable. Fetal heart tones were 135 beats per minute. The fetus was in the cephalic presentation.

An electrocardiogram (EKG) was performed immediately which revealed ST segment elevations in leads V2-V4 (Figure 1). A bedside echocardiogram was performed which revealed anterior wall and left ventricular apical wall motion abnormalities. The diagnosis of a myocardial infarction or spontaneous dissection of a coronary artery was made by the cardiology team. Maternal Fetal Medicine (MFM) and Cardiology consulted and agreed that cardiac catheterization was appropriate.

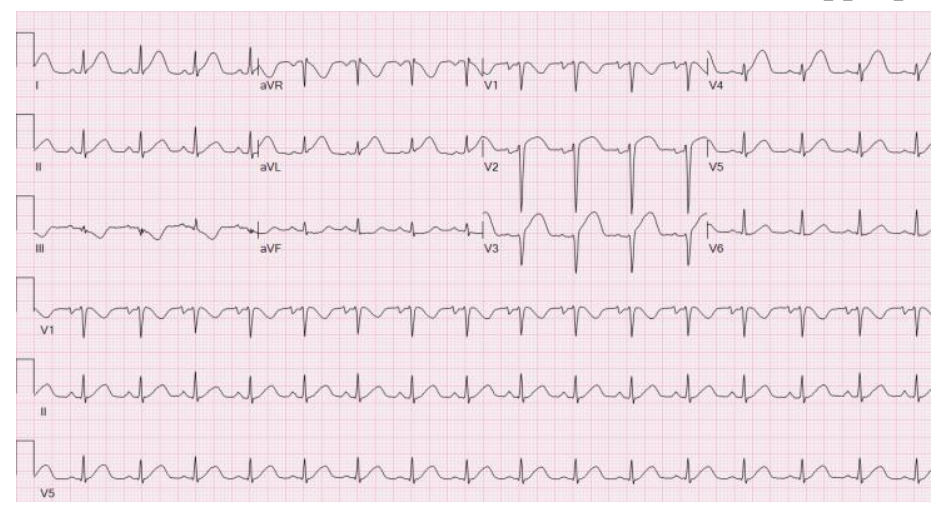

Figure1. Electrocardiogram in the emergency room indicating ST segment elevations in leads V2-V4

The patient was immediately taken to the cardiac catheterization laboratory where a cardiac catheterization revealed a spontaneous coronary artery dissection of the left anterior descending artery with a large intramural hematoma that extended to the first $1 / 3$ of the left anterior descending artery (Figure 2). Given these findings, interventional cardiology recommended against coronary artery stent placement due to concern for extension of the dissection past the stent.

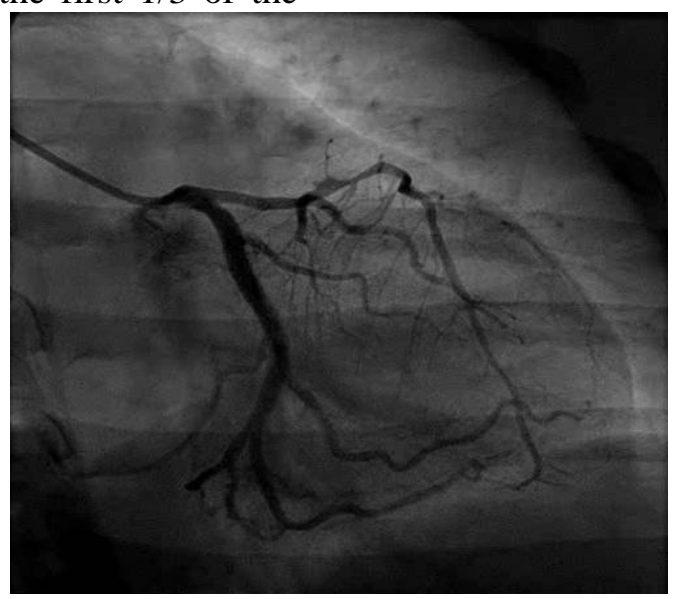

Figure2.Cardiac catheterization upon initial presentation showing spontaneous coronary artery dissection of the left anterior descending artery. 
The patient remained hemodynamically stable and did not require emergent coronary artery bypass graft surgery. The patient was transferred to the cardiac intensive care unit (CCU) for medical management of her coronary artery dissection. In consultation with MFM the patient was treated with intravenous nitroglycerin, heparin, beta blockade and one dose of intravenous Plavix. Throughout this entire time the obstetric team was present at the bedside and prepared to perform an emergency cesarean section should maternal cardiac arrest occur or if fetal heart rate abnormalities were to be seen. The patient had multiple bouts of short lived ventricular tachycardia which resolved with administration of digoxin.

After the patient's cardiac status remained stable with medical management for three days, a multidisciplinary meeting was held including representatives from Anesthesia, Cardiothoracic surgery, Interventional cardiology, CCU, and Maternal Fetal Medicine. Multiple scenarios were discussed. Maternal Fetal Medicine suggested that the patient be allowed to labor with the plan for a dense epidural and expedited seconded stage by instrument delivery. The cardiology and anesthesia teams were very concerned that even the smallest amount of pain or pressure could cause the patient to inadvertently Valsalva, leading to extension of the coronary artery dissection, or cause another episode of ventricular tachycardia. They also felt a cesarean section in the main Cardiac OR would afford them the most support if an acute cardiac event were to occur. Despite the patient's parity and history of precipitous deliveries in the past, as well as her being 39 weeks gestation the decision was made to proceed with the Cesarean section in the cardiovascular OR. This was done so that cardiology and cardiothoracic surgery support would be immediately available if a cardiac event were to occur during the delivery process.

A Cesarean section was performed under general anesthesia in the cardiac operating room. An arterial line and a central line were placed by anesthesia prior to the cesarean section to monitor maternal hemodynamic state intraoperatively. Cardiothoracic surgery was present in the event that an emergent CABG were to be required. Cardiology was available in the event an arrhythmia were to develop. Twenty minutes after intubation, after all lines were secured, the patient was delivered via cesarean section of a female neonate weighing $3160 \mathrm{~g}$. The cesarean section was accomplished without complications. There was no excessive bleeding despite her being treated with Plavix. The neonate required intubation secondary to maternal general anesthesia prior to delivery and was extubated one hour later. The arterial cord blood gases revealed: $\mathrm{pH} 7.24, \mathrm{pCO} 266, \mathrm{BE}-$ 2.3. The estimated blood loss during delivery was $700 \mathrm{cc}$. The surgery was well tolerated without any cardiac events intraoperatively.

Post operatively the patient was extubated immediately and taken to the CCU for her recovery period. A repeat echocardiogram showed severe hypokinesis of the mid to distal anterior wall, antero-septum, distal anterolateral wall and apex of the left ventricle consistent with an infarct along the left anterior descending artery. The ejection fraction was 30$35 \%$. Post operatively the patient remained hemodynamically stable. On the fourth postoperative day the patient had a repeat cardiac catheterization (Figure 3). This revealed findings consistent with a healing spontaneous coronary artery dissection. The patient was discharged home on post-operative day five with a life vest and medications.

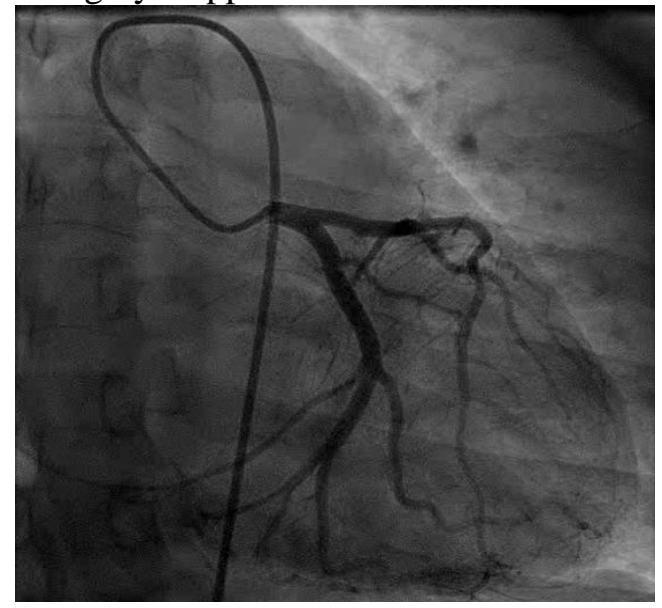

Figure3. Cardiac catheterization on post-operative day four after cesarean section showing healing spontaneous coronary artery dissection of the left anterior descending artery. 
At 2 and 6 weeks post-operatively the patient was seen by cardiology and obstetrics for follow up. She was asymptomatic. Her incision was well healed, she was bottle feeding effectively, and her vaginal bleeding was minimal. She was found to have postpartum depression and was started on Zoloft with a referral for psychiatric care. Her cardiac function was stable on Lisinopril, aldactone, metoprolol, aspirin and Plavix. No arrhythmic events were detected on her life vest. The plan was to repeat the echocardiogram 3 months post-operatively to assess left ventricular ejection fraction and determine if an ICD were to be needed for primary prevention of sudden cardiac events.

\section{DISCUSSION}

Spontaneous coronary artery dissection is a rare cause of myocardial infarction among the general population; however, it seems to have a propensity for the pregnant and postpartum patient population. It has been noted to mostly affect multiparous women over the age of thirty ${ }^{13}$ and commonly involves the left main and left anterior descending arteries ${ }^{14}$. The exact mechanism for this is unknown; however, it is thought that the increased level of progesterone results in structural changes of the vessel walls making them more susceptible to spontaneous dissection ${ }^{15-16}$. With the increased hemodynamic stress of pregnancy and delivery, small tears in the intima of these compromised vessels can occur leading to the dramatic clinical picture that occurs ${ }^{16}$.

There appears to be a consensus in the literature that conservative medical management is the most appropriate mode of treatment for clinically stable patients ${ }^{4,11-13,17}$. In contrast to this, there is very little data on the best mode of delivery for patients who have suffered from SCAD during the pregnancy. This may be because majority of these cases occur in the postpartum period after delivery has occurred. However, there are cases where patients present with SCAD during the antepartum period and mode of delivery needs to be addressed.

Pregnancy is associated with certain changes in physiology that can worsen any heart condition. There is an increase in blood volume, stroke volume and heart rate which causes an increase in myocardial oxygen demand. At the same time, there is a decrease in diastolic blood pressure and a physiologic anemia that occurs which causes a decrease in myocardial oxygen supply. For the young, healthy woman without other medical comorbidities, the physiologic changes of pregnancy are well tolerated. However, in a woman with an underlying cardiac condition, these changes can lead to exacerbation of any cardiac condition ${ }^{18}$. During labor, the combination of pain, anxiety and contractions causes an even further increase in maternal heart rate, stroke volume, cardiac output and blood pressure. Additionally, after delivery of the placenta there is a $500 \mathrm{cc}$ autotransfusion that occurs which may not be tolerated by the patient with cardiac decompensation $^{21}$. It is recommended that delivery be postponed at least two weeks from the time of infarction if possible to allow for some amount of cardiac healing after the ischemic event ${ }^{4},{ }^{18-20}$. Maternal mortality is higher if delivery is sooner than two weeks from the ischemic event, possibly due to increase in hemodynamic requirements during labor which increase the myocardial demand and the risk of recurrent myocardial ischemia in a heart that has not had sufficient time to heal from the initial ischemic event ${ }^{18-20}$.

When determining appropriate mode of delivery, consideration must be given to determine what will have the least hemodynamic burden. Recommendations from many years of experience with cardiac patients who are pregnant have focused on decreasing the risk to the mother during delivery. Early epidural placement is recommended for all women with cardiac history as it will decrease the sympathetic stimulation in response to pain, thereby decreasing myocardial oxygen consumption during labor ${ }^{4}$ 18, 21-22. The second stage of labor is associated with an increase in hemodynamic and oxidative cardiac stress, likely secondary to strenuous Valsalva necessary for fetal expulsion ${ }^{4,18,21}$. Operative vaginal delivery and a shortened second stage will reduce the amount of Valsalva necessary for delivery, thereby reducing the oxidative stress on the heart ${ }^{4,18,21,23}$. Cesarean section, on the other hand, is associated with an increased risk of hemorrhage, infection and large fluid shifts $^{21}$. Whereas the risk of increase oxidative stress on the heart secondary to Valsalva with a vaginal delivery can be avoided with use of forceps or vacuum to shorten the second stage, the risks that will occur with a cesarean section are unavoidable. Therefore, the optimal mode of delivery for most patients with cardiac disease would be an operative vaginal delivery to shorten the second stage rather than a cesarean section ${ }^{4}$. 
After the multidisciplinary meeting and knowing the unpredictable nature of extension of coronary artery dissection as well as the possibility to need advance surgical and extracorporeal support, the team questioned the applicability of the "standard" approach to delivery of patients with heart disease who are pregnant. With limited data to guide us, the decision for cesarean section was made based on maximizing the resources available to care for a sudden cardiac event during labor or delivery. Cesarean section affords the patient at term and all disciplines involved the best resources needed should a cardiac event ensue. This approach may not be appropriate if there is a patient that experiences SCAD earlier in the third trimester and recovers after medical management with sufficient time for cardiac healing. In those cases a vaginal delivery with a dense epidural and expedited second stage of labor may offer the less stress on the heart and a better prognosis postpartum.

We believe from this case that the standard approach to labor and delivery for patients with cardiac disease may not apply to patients with SCAD. We hope that this case can stimulate further research into the best approach to delivery for all cardiac patients who are at risk of acute decompensation given the modern advances and resources now available to treat these events.

\section{REFERENCES}

[1] Elkayam U, Jalnapurkar S, Barakkat MN, Khatri N, Kealey AJ, Mehra A, et al. Pregnancy-associated acute myocardial infarction: a review of contemporary experience in 150 cases between 2006 and 2011. Circulation 2014;129:1695-702

[2] Thompson EA, Ferraris S, Gress T, Ferraris V. Gender differences and predictors of mortality in spontaneous coronary artery dissection: a review of reported cases. $\mathrm{J}$ Invasive Cardiol2005;17:59-61

[3] Tweet MS, Hayes SN, Pitta SR, Simari RD, Lerman A, Lennon RJ, et al. Clinical features, management, and prognosis of spontaneous coronary artery dissection. Circulation 2012;126:579-88

[4] Codsi E, Tweet MS, Rose CH, Arendt KW, Best PJM, Hayes SN. Spontaneous coronary artery dissection in pregnancy: what every obstetrician should know. Obstetrics and Gynecology 2016;128:731-8

[5] Becker RM. Intracardiac surgery in pregnant women. Ann ThoracSurg1983;36:453-8
[6] Bernal JM, Miralles OJ. Cardiac surgery with cardiopulmonary bypass during pregnancy. ObstetGynecolSurv1986;41:1-6

[7] John AS Gurley F, Schaff HV, Warnes CA, Phillips SD, Arendt KW, et al. Cardiopulmonary bypass during pregnancy. Ann ThracSurg2011;;9:1191-6

[8] Pomini F, Mercogliano D, Cavalletti C, Caruso A, Pomini P. Cardiopulmonary bypass in pregnancy. Ann ThracSurg1996;61:259-68

[9] Strickland Ra, Oliver WC Jr, Chantigian RC, Ney JA, Danielson GK. Anesthesia, cardiopulmonary bypass, and the pregnancy patient. Mayo ClinProc 1991;66:411-29

[10] Weiss BM, von Segesser LK, Alon E, Seifert B, Turina MI. Outcome of cardiovascular surgery and pregnancy: a systematic review of the period 1984-1996. Am J ObstetGynecol1998;179:1643-5

[11] Havakuk O, Goland S, Mehra A, Elkayam U. Pregnancy and the risk of spontaneous coronary artery dissection: an analysis of 120 contemporary cases. CircCardiovascInterv 2017;10:e004941

[12] Tweet MS, Eleid MF, Best PJ, Lennon RJ, Lerman A, Rihal CS, et al. Spontaneous coronary artery dissection: revascularization versus conservative therapy. CircCardiovascInterv2014;7:777-86

[13] Maeder M, Ammann P, Angehrn W, Rickli H. Idiopathic spontaneous coronary artery dissection: incidence, diagnosis and treatment. Int J Cardiol2005;101:363-9

[14] Gonzalez Amieva A, Lostal Navarro C, Gutierrez Solis MA, SachezTejero E, Garcia Sanchez JE. Acute myocardial infarction in a pregnant woman due to coronary artery dissection. Ann Med Interna2002;19:544-45

[15] Manalo-Estrella P, Barker AB. Histopathologic findings in human aortic media associated with pregnancy. Arch Pathol Lab Med 1967;83:33641

[16] Bonnet J, Aumailley M, Thomas D, Grosgogeat Y, Broustet JP, Bricaud H. Spontaneous coronary artery dissection: case report and evidence for a defect in collagen metabolism. Eur Heart J 1986;7:904-9

[17] Appleby CE, Barolet A, Ing D, Ross J, Schwardz L, Seidelin P, Silversides C, Horlick E. Contemporary management of pregnancyrelated coronary artery dissection: A singlecentre experience and literature review. ExpClinCardiol 2009;14:e8-e26

[18] Kealey AJ. Coronary artery disease and myocardial infarction in pregnancy: A revie of epidemiology, diagnosis and medical and surgical management. Can J Cardiol 2010;26:e185-e189 
[19] Roth A, Elkayam U. Acute myocardial infarction associated with pregnancy. Ann Intern Med 1996;125:751-62

[20] Hankins GD, Wendel GD Jr, Leveno KJ, Stoneham J. Myocardial infarction during pregnancy: a review. ObstetGynecol1985;65:139-46

[21] Simpson LL. Maternal cardiac disease: update for the clinician. Obstetrics and Gynecology 2012;199:345-59
[22] Kuczkoski KM. Labor analgesia for the parturient with cardiac disease: what does an obstetrician need to know? ActaObstetGynecolScand2004;8:223-33

[23] Dufour P, Berard J, Vinatier D, Subtil D, Guinet B, Bourzoufi K, Michon P, Puech F. Pregnancy after myocardial infarction and a coronary artery bypass graft. Arch GynecolObstet1997;259:209-13

Citation: Cheryl Yondorf, Arnold Cohen, Mode of Delivery for a Term Patient with Spontaneous Coronary Artery Dissection. ARC Journal of Gynecology and Obstetrics. 2018; 3(1):1-6. DOI: dx.doi.org/10.20431/2456-0561.0301001.

Copyright:@ 2018 Authors. This is an open-access article distributed under the terms of the Creative Commons Attribution License, which permits unrestricted use, distribution, and reproduction in any medium, provided the original author and source are credited. 